\title{
Nach der Erziehung zur Mündigkeit. Anmerkungen zu Antisemitismus und Pädagogik
}

\author{
Michael Fischer
}

An einem spätherbstlichen Abend im November 2017 riefen einige hundert Menschen wütende Parolen in Richtung der US-Botschaft in Wien. Mitgebracht hatten die Demonstranten neben Palästina- und Türkeifahnen auch den Hass auf den israelischen Staat. Anlass für die Versammlung war die angekündigte Verlegung der US-Botschaft in Israel von Tel Aviv nach Jerusalem. ${ }^{1}$ An der Lage der Menschen in Israel und Palästina änderte diese symbolische Aktion der Vereinigten Staaten, die inzwischen rechtlich umgesetzt wurde, de facto rein gar nichts. Jerusalem war ohnedies bereits die israelische Hauptstadt, auch wenn die meisten arabischen und islamischen Staaten diese Tatsache, wie auch insgesamt die Existenz eines souveränen jüdischen Staates nicht anerkennen wollen. Der türkische Präsident Tayyip Recep Erdogan ergriff Ende 2017 dennoch die Chance und setzte sich rhetorisch an die Spitze einer Bewegung empörter Muslime. Niemals werde er die Entscheidung der USA anerkennen, verlautete Erdogan. Mit ihr habe Donald Trump Jerusalem zum Kerker für Muslime und andere Religionen gemacht. Der Kampf der Muslime werde erst enden, wenn ein palästinensischer Staat errichtet sei, so Erdogan. ${ }^{2}$

In Erdogans Rede und den Botschaften der antiisraelischen Demonstranten bricht sich ein irrationaler Erlösungsglaube Bahn, der in Israel das alleinige Hindernis für den Weltfrieden zu erkennen glaubt. Friede auf der gesamten Welt gibt es in diesem teleologischen Weltbild erst dann, wenn ein eigener palästinensischer Staat gegründet worden ist: ein großpalästinensischer Staat - nicht neben, sondern anstelle des israelischen versteht sich -, ein Staat, in dem Juden bestenfalls noch als Minderheit geduldet würden. Die bestehenden Probleme der islamischen Welt, wie unzählige interne und externe bewaffnete Konflikte, Bürgerkrieg, islamischer Radikalismus, wirtschaftliche und gesellschaftliche Stagnation würden selbstverständlich auch nach Errichtung eines solchen Staates weiterbestehen, doch die Frage um den Status von Jerusalem scheint offensichtlich mehr Menschen zu bewegen, als die Lösung solch weltlicher und ganz realer Probleme. Statt über die Fehler und Probleme der eigenen Gesellschaft zu reflektieren, erkennt man in Israel den Schuldigen.

\footnotetext{
${ }^{1}$ Die Botschaft wurde inzwischen offiziell nach Jerusalem verlegt. De facto wurde dazu das dortige Generalkonsulat umbenannt, während die meisten Einrichtungen der Botschaft nach wie vor in Tel Aviv sind. Siehe auch U.S. Embassy in Israel, o. D., [https://il.usembassy.gov/], eingesehen 1.6.2020.

${ }^{2}$ Jerusalem-Streit, Erdogan gibt USA Mitschuld an Gewalt, in: Die Zeit, 11.12.2017,

[http://www.zeit.de/politik/ausland/2017-12/jerusalem-streit-recep-tayyip-erdogan-israel-usa], eingesehen 1.6.2020.
} 
Man kennt diese Reaktion aus der Geschichte des Antisemitismus. Für Theodor W. Adorno und Max Horkheimer beruht der Antisemitismus auf falscher Projektion: Regungen in einem selbst, die nicht als die eigenen akzeptiert werden können, werden jemand anderem zugeschrieben; dem, der nun zum Opfer wird. ${ }^{3}$ Das Subjekt verliert die Fähigkeit zur Reflexion in zwei Richtungen: „,[...] da es nicht mehr den Gegenstand reflektiert, reflektiert es nicht mehr auf sich und verliert so die Fähigkeit zur Differenz. Anstatt der Stimme des Gewissens hört es Stimmen; anstatt in sich zu gehen, um das Protokoll der eigenen Machtgier aufzunehmen, schreibt er die Protokolle der Weisen von Zion den anderen zu. "“4

Anhand Erdogans Rede und der Rhetorik seiner Sympathisanten in Wien, die vor der US-Botschaft demonstrierten, lässt sich dieses simple Projektionsmuster nachzeichnen: Israel wird vorgeworfen, an einem Großreich zu arbeiten, dabei hängen der türkische Präsident und viele seiner Anhänger selbst dem Traum einer imperialistischen neoosmanischen Ideologie an. ${ }^{5}$ Man wirft Israel „Kindermord“ vor, während die türkische Armee Kriegsverbrechen begeht und kurdische Dörfer bombardiert. ${ }^{6}$

Nicht nur in Israel werden Juden immer wieder Opfer der islamischen „Israel-Kritik“, die sich dort regelmäßig wiederkehrend in Messer- oder Bombenattentaten äußert. Auch in Schweden wurde die sogenannte „Kritik“ im Dezember 2017 praktisch: Jugendliche warfen Brandsätze auf eine Synagoge in Göteborg. ${ }^{7}$ Auch hier wurden in den Medien Zusammenhänge mit den Protesten gegen die Botschaftsverlegung der USA thematisiert, die in den Tagen zuvor in vielen schwedischen Städten abgehalten worden waren. In Malmö hatte sich diese „Kritik“ an der Botschaftsverlegung in antisemitischen Parolen geäußert, wie die Tageszeitung Die Welt berichtete. ${ }^{8}$

Zur Rechtfertigung derartiger Hass- und Gewalttaten werden die Juden und Israel als besonders mächtig imaginiert, um mit gutem Gewissen zuschlagen zu können. Das Opfer wird zum Täter umgedeutet: „Stets hat der blind Mordlustige im Opfer den Verfolger gesehen, von dem er verzweifelt sich zur Notwehr treiben ließ, und die mächtigsten Reiche haben den schwächsten Nachbarn als unerträgliche Bedrohung empfunden, ehe sie über ihn herfielen." In Wien blieb am eingangs beschriebenen Novemberabend alles ruhig, vermeldete die Polizei. Laut Zeugenaussagen skandierte die

\footnotetext{
${ }^{3}$ Max Horkheimer/Theodor W. Adorno, Dialektik der Aufklärung, in: Max Horkheimer, Gesammelte Schriften, Band 5. Dialektik der Aufklärung und Schriften 1940-1950, Frankfurt a. M. 2003, S. 217.

${ }^{4}$ Horkheimer/Adorno, Dialektik der Aufklärung, S. 219-220.

${ }_{5}^{5}$ Siehe bspw. Mustafa Türkeş, Decomposing Neo-Ottoman Hegemony, in: Journal of Balkan and Near Easter Studies 18 (2016), Heft 3, S. 191-216.

${ }^{6}$ Amnesty wirft Türkei Kriegsverbrechen vor, in: tagesschau.de, 18.10.2019, [https://www.tagesschau.de/ausland/amnesty-tuerkei-kriegsverbrechen-101.html], eingesehen 4.6.2020.

${ }^{7}$ Maskierte werfen Molotowcocktails auf Synagoge in Göteborg, in: Die Welt, 10.12.2017, [https://www.welt.de/politik/ausland/article171441557/Maskierte-werfen-Molotowcocktails-auf-Synagoge-inGoeteborg.html], eingesehen 11.12.2017.

8 Ebd.

${ }^{9}$ Horkheimer/Adorno, Dialektik der Aufklärung, S. 219-220.
} 
Menge jedoch „Schlachtet die Juden“. Es blieb der Wiener FPÖ überlassen, eine Anzeige bei der Staatsanwaltschaft einzubringen. ${ }^{10}$

Mit dieser hier skizzierten Konstellation ist auch schon ein Dilemma beschrieben, in dem sich all jene wiederfinden, die sowohl Fremdenfeindlichkeit als auch den Antisemitismus ablehnen. Weil die meist türkischen und arabischen Demonstranten in Wien selbst fremdenfeindlich diskriminiert werden, scheut man im linken oder progressiven Milieu vielfach vor der Kritik am islamischen Antisemitismus zurück. Das ist einerseits ein Schweigen aus taktischen Überlegungen, freilich sind andererseits Teile der Linken in Form der „Israel-Kritik“ ihrerseits dieser Spielart des Antisemitismus verfallen. ${ }^{11}$ Wie es sich im Einzelnen auch verhält: Die Kritik des islamischen Antisemitismus gilt als Thema, welches man in der Öffentlichkeit besser nicht anspricht. Oft besteht die Angst, der FPÖ bzw. der Rechten damit argumentative Munition zu liefern oder die latente Fremdenfeindlichkeit in der Gesellschaft zu befeuern. Dabei weiß in Österreich, nicht zuletzt durch mediale Berichterstattung, ${ }^{12}$ bereits jetzt schon fast jeder über die islamische Judenfeindschaft Bescheid und diese Thematisierung wird wohl kaum jemand zum Fremdenfeind machen, der es nicht schon davor war. Fremdenfeindlichkeit äußert sich schließlich nicht in der Einstellung zu Ideologien wie dem Islam oder im Grad der Ablehnung des islamischen Antisemitismus, sie äußert sich in einem biologistischen und essentialistischen Menschenbild. ${ }^{13}$ In diesem sind Menschen durch ihre Geburt an eine bestimmte Kultur gebunden. Für einen Fremdenfeind ist ein Muslim folglich von Geburt an Muslim und dieses Charakteristikum ist auch nicht veränderbar. Wer jedoch nicht von dieser Unveränderlichkeit der Menschen ausgeht, wer nicht davon ausgeht, dass die Kultur angeboren ist, hegt die Hoffnung, die Kritik könnte manche vom Antisemitismus abbringen. Die Kritik des islamischen Antisemitismus ist deshalb auch ein notwendiges Mittel, um das friedliche Zusammenleben der Menschen in Österreich zu garantieren.

\section{Antisemitismus bei muslimischen Jugendlichen}

In den letzten Jahren beschäftigten sich mehrere Studien in Österreich implizit oder explizit mit dem islamischen Antisemitismus. ${ }^{14}$ So wollten Georg Lauss und Stefan

10 Zeugen geschockt über „Schlachtet die Juden“-Ruf, in: Heute, 9.12.2017,

[http://www.heute.at/oesterreich/wien/story/Zeugen-geschockt-ueber--Schlachtet-die-Juden--Ruf-48060169], eingesehen 11.12.2017.

11 Zum linken Antisemitismus siehe David Hirsh, Contemporary Left Antisemitism, Abingdon-on-Thames 2017. Vergleiche auch den Beitrag von Daniel Rickenbacher zu links-islamistischen Allianzen in diesem Band.

12 Siehe bspw. Merkel räumt Problem des Antisemitismus unter Geflüchteten ein, in: Der Standard, 22.4.2018, [https://www.derstandard.at/story/2000078433573/merkel-raeumt-problem-des-antisemitismus-untergefluechteten-ein], eingesehen 1.6.2020.

13 Siehe bspw. Anti-Defamation League, Racism, [https://www.adl.org/racism], eingesehen 1.6.2020.

${ }^{14}$ Eine Überblicksstudie über die Situation in mehreren europäischen Ländern bietet: Günther Jikeli, European Muslim Antisemitism. Why Young Urban Males Say They Don't Like Jews, Bloomington 2015. 
Schmid-Heher in zwei Studien wissen, wie groß die Zustimmung zum demokratischen System unter Wiener Berufsschülern und Lehramtsstudenten ist. ${ }^{15}$ Von beiden Studien wurde bisher nur eine vollständig veröffentlicht. ${ }^{16}$ Zumindest in der Studie zu den Lehramtsstudenten finden sich einige diskussionswürdige Abschnitte.

Im Teilbereich „Politischer Autoritarismus“ wurden die Teilnehmer mit folgender Aussage konfrontiert: „Die Demokratie ist die beste Regierungsform, auch wenn sie Probleme mit sich bringen mag. " 17 Wer dies verneinte bzw. ablehnte, wurde in der Auswertung dem politischen Autoritarismus zugeschlagen. Selbst wenn mit der Ablehnung gemeint gewesen sein könnte - was als mögliche Antwort durchaus plausibel erscheint -, eine noch menschenfreundlichere Form des gesellschaftlichen Zusammenlebens sei denkmöglich. Für die Auswertung der Studie galt den Autoren allerdings nur die Affirmation der bestehenden Verhältnisse als progressiv. Im Sinne Adornos würde eine derartige Affirmation hingegen eher auf einen autoritären Charakter hinweisen. ${ }^{18}$ Auch die Zukunftsangst, die bei der aktuellen Weltlage vielmehr eine realistische Einschätzung der gesellschaftlichen Entwicklung ist, als eine unbegründete Angst, wird in dieser Studie letztlich zum Indikator für Autoritarismus. Zudem nimmt die Studie theoretisch Bezug auf Denktraditionen, die inhaltlich wenig gemeinsam haben und nicht einfach unwidersprochen nebeneinanderstehen können. Adorno und Karl Popper werden gleichzeitig bemüht, doch beide waren im Positivismusstreit der deutschen Soziologie nicht ohne Grund Widersacher. ${ }^{19}$

Die zweite Studie über die politischen und gesellschaftlichen Einstellungen der Berufsschüler in Wien ist noch unveröffentlicht. ${ }^{20}$ Einige Ergebnisse drangen durch einen Artikel der Tageszeitung Der Standard jedoch bereits 2017 an die Öffentlichkeit. Dieser titelte: „Starke antisemitische Vorurteile bei muslimischen Jugendlichen. “21 Eine Frage, die Lauss und Schmid-Heher den Probanden stellten, war, ob die Juden in Österreich zu viel Einfluss hätten. Dem stimmten $24 \%$ der Befragten zu. Ein nicht zu unterschätzend hoher Prozentsatz. Noch höher ist der Glaube an den zu großen Einfluss der Juden in Österreich bei Berufsschülern, die zuhause türkisch, bosnisch, albanisch

\footnotetext{
15 Vergleiche den Beitrag der Autoren in diesem Sammelband.

16 Georg Lauss/Stefan Schmid-Heher, Politische Bildung und demokratierelevante Einstellungsmuster von angehenden Lehrenden. Eine quantitative Studie, in: R\&E-SOURCE (2017), [https://journal.phnoe.ac.at/index.php/resource/article/view/350], eingesehen 11.12.2017, S. 5.

17 Ebd., S. 5.

18 Theodor W. Adorno u. a., The Authoritarian Personality, New York 1950.

19 Hans-Joachim Dahms, Der Positivismusstreit der 60er Jahre: eine merkwürdige Neuauflage, in: Jahrbuch für Soziologiegeschichte 1991, Opladen 1992, S. 119-182.

${ }^{20}$ Inzwischen ist ein Pre-Print über Research Gate abrufbar: Georg Lauss/Stefan Schmid-Heher, Politische Bildung an Wiener Berufsschulen: Demokratische und autoritäre Potentiale von Lehrlingen, Wien 2017 [https://www.researchgate.net/publication/324755324_Politische_Bildung_an_Wiener_Berufsschulen_Demokratis che_und_autoritare_Potentiale_von_Lehrlingen], eingesehen 1.6.2020.

21 András Szigetvari, Starke antisemitische Vorurteile bei muslimischen Jugendlichen, in: Der Standard, 17.6.2017, [https://www.derstandard.at/story/2000059359305/starke-antisemitischen-vorurteile-bei-muslimischenjugendlichen], eingesehen 1.6.2020.
} 
oder arabisch sprechen. Dort vertraten $48 \%$ der Befragten dieses Weltbild. Sehr subtil wurde hier von den beiden Studien-Autoren nicht nach dem Antisemitismus gefragt. ${ }^{22}$ Die Zustimmungsraten bei derartig offen antisemitischen Aussagen sind in der Regel gering. In der Mehrheitsgesellschaft ist ein solch offen vorgetragener Antisemitismus verpönt. Hier greift man stattdessen mit gutem Gewissen zur „Israel-Kritik“, beschimpft den jüdischen Staat pseudo-antifaschistisch als rassistisch und ruft zum Boykott israelischer Waren auf. ${ }^{23}$ Personen, die sich selbst als progressiv sehen, fasziniert von fremden Kulturen sind und einen Hang zu den beschriebenen antisemitischen Denkmustern aufweisen, werden diese Frage nach der Macht der Juden höchstwahrscheinlich negativ beantworten. $\mathrm{Zu}$ sehr klingt sie nach klassisch nationalsozialistischem Antisemitismus, der in der Regel abgelehnt wird. Umso verwunderlicher sind die $48 \%$ Zustimmung bei jenen Kindern, bei denen die Eltern auch eine andere Sprache als Deutsch sprechen. Auch bei ihnen muss man von einem Hang zur gesellschaftskonformen Beantwortung der Frage ausgehen. Doch dürfte der Bezugsrahmen nicht die österreichische Mehrheitsgesellschaft sein, sondern vielfach ein islamisch-konservatives Milieu. Vieles deutet deshalb auf eine hohe Verbreitung antisemitischer Denkmuster in diesem gesellschaftlichen Umfeld hin. Denn trotz der recht mageren Datenmenge ist eine Tendenz klar zu erkennen.

Wie sehr der Antisemitismus speziell unter muslimischen Berufsschülern verbreitet ist, kann aus diesen Daten jedoch letztlich nicht herausgelesen werden. Abgefragt wurde nur die „Muttersprache“ und nicht die Religionszugehörigkeit. Doch von der Muttersprache kann selbstverständlich nicht darauf geschlossen werden, wie es jemand mit der Religion bzw. dem Islam hält. ${ }^{24}$ Es würde folglich nicht überraschen, wenn der Antisemitismus unter islamisch-religiösen Jugendlichen in dieser Gruppe der „Nicht-DeutschenMuttersprachler" deutlich höher wäre, als unter tendenziell nichtreligiösen und säkularen bzw. Nichtmuslimen, die sich in der Gruppe ebenfalls finden dürften. Die Studie gibt darauf allerdings keine Antwort. Diese Tendenz lässt sich zumindest aus einer Studie des Soziologen Kenan Güngör herleiten. ${ }^{25}$ Sie hatte die Einstellung zu gesellschaftlichen Fragen der Jugendlichen in Wiener Jugendhäusern zum Gegenstand und wurde 2016 durchgeführt. Ein Drittel der befragten Jugendlichen zeigte gegenüber Juden negative Einstellungen. Nach Religionszugehörigkeit ergab sich folgendes Bild: $47 \%$ der Muslime

22 Ähnlich plump fragt die Autoritarismusstudie der Österreichischen Hochschülerschaft nach dem Antisemitismus: „Wenn es zu Auswüchsen des Kapitalismus kommt, dann sind daran meistens die Juden/Jüdinnen Schuld“, ist das entsprechende Item in deren Studie von 2018. Petra Ziegler, Befragung von Studierenden in Österreich zu Autoritarismus, Geschichtsbildern und demokratischer Disposition, Wien 2018, S. 31, [https://www.oeh.ac.at/file/2086/download?token=nt9ZraU9], eingesehen 1.6.2020.

23 Siehe bspw. Bildungsstätte Anne Frank (Hrsg.), 4 Grüne, warum BDS antisemitisch ist, [https://www.bs-annefrank.de/fileadmin/user_upload/Slider/Publikationen/BDS_Kritik_Broschuere.pdf], eingesehen 1.6.2020.

${ }^{24}$ In der Medienberichterstattung, u. a. im Standard, wurde auf diese Diskrepanz allerdings keine Rücksicht genommen. ${ }^{25}$ Kenan Güngör/Caroline Nik Nafs, Jugendliche in der offenen Jugendarbeit. Identitäten, Lebenslagen \& abwertende Einstellungen, Wien 2016, [https://www.wien.gv.at/freizeit/bildungjugend/pdf/studie-1.pdf], eingesehen 11.12.2017. 
lehnten Juden ab, bei den Orthodoxen Christen waren es $27 \%$ und unter den Katholiken waren es $7 \%$.

Für Güngör speist sich dieser islamische Antisemitismus vor allem aus der Rezeption des Nahostkonflikts. Als Muslime solidarisierten sie sich mit ihren „Brüdern und Schwestern“ in Palästina. Bei vielen Menschen im und aus dem Nahen Osten herrsche die Vorstellung vor, der Westen habe sie immer schon unterdrückt und die Speerspitze dieser westlichen Unterdrückung sei Israel. In den meisten arabischen Medien erhalte Israel den Platz des globalen Bösewichts. Ein Unterschied zwischen Juden und Israel werde dabei nicht gemacht. Diese Sendungen würden auch in Österreich über Onlineplattformen wie YouTube oder Satellitenfernsehen konsumiert. Viele Jugendliche aus islamischen Familien hätten folglich das Gefühl, es sei gesellschaftlich akzeptiert, antisemitische Einstellungen zu vertreten. Jugendliche aus der Mehrheitsbevölkerung sind hier dagegen schon vorsichtiger. ${ }^{26}$ Der Hang dazu, Fragen gesellschaftlich erwünscht zu beantworten, könnte auch hier eine Erklärung des großen Unterschieds zwischen Katholiken und Muslimen sein. Nämlich die unterschiedlichen sozialen Referenzrahmen. Den massiven Unterschied von gleich $40 \%$ kann das jedoch allein nicht erklären. Für Güngör ist dieser islamische Antisemitismus deshalb so gefährlich, weil er mit dem israelisch-arabischen Konflikt in Verbindung steht. Immer wenn in Nahost etwas passiere, flamme auch der Antisemitismus in Österreich auf.

Dementsprechend wird der islamische Antisemitismus in naher Zukunft wahrscheinlich auch ein drängenderes Problem werden. Es gibt immer mehr Schüler mit Migrationshintergrund in den Klassenzimmern und 2015 kamen viele neue Menschen aus Syrien, dem Irak und Afghanistan nach Österreich. In diesen Ländern wird und wurde der Antisemitismus lange Zeit politisch eingesetzt und staatlich gefördert. Es wäre also ein Wunder, wenn 2015 nicht auch Menschen nach Österreich kamen, die antisemitischen Welterklärungen anhängen. ${ }^{27}$

\section{Die spezifische Verantwortung als Österreicher}

Der Wiener Zeithistoriker Oliver Rathkolb empfahl als Reaktion auf die genannten Studienergebnisse bereits im Kindergarten und in der Volksschule Maßnahmen gegen Vorurteile zu ergreifen. Ein Fokus solle dabei auf die Berufsschulen gelegt werden, diese seien bisher vergessen worden. Dabei besuchen beinahe $40 \%$ der 15-Jährigen eine solche Lehranstalt. Gerade in Wien hätten viele Lehrlinge einen sogenannten Migrationshintergrund. In der Schule werden Antisemitismus und der Holocaust bislang meist gleichzeitig behandelt. Darin liegt für Rathkolb ein Problem, weil sich diese Art des Unterrichts explizit an die Nachfahren der Täter des Holocausts richte. Diese

${ }^{26}$ Szigetvari, Starke antisemitische Vorurteile.

${ }^{27}$ Siehe dazu auch den Beitrag von Günther Jikeli in diesem Band. 
„Betroffenheitspädagogik“ werde Schulklassen auch in Gedenkstätten, wie dem Mauthausen Memorial, vermittelt. Türkische und arabische Jugendliche erreiche man damit nicht, für sie sei der Holocaust eine europäische Angelegenheit und damit hätten sie nichts zu schaffen. ${ }^{28}$ Damit spricht Rathkolb einen wichtigen Punkt an. Die Aufklärung über den Antisemitismus darf nicht auf Betroffenheit oder einem schlechten Gewissen aufbauen. Denn auch Schülerinnen und Schüler, die sich selbst als Österreicher verstehen, wird man damit kaum noch nachhaltig erreichen.

Aufgrund der spezifischen österreichischen Geschichte müsse man entschieden gegen Antisemitismus und Rassismus vorgehen, forderte noch im November 2017 die damals scheidende Bildungsministerin Sonja Hammerschmid von der SPÖ. ${ }^{29}$ Der kategorische Imperativ Adornos lautete dagegen: „Hitler hat den Menschen im Stande ihrer Unfreiheit einen neuen kategorischen Imperativ aufgezwungen: ihr Denken und Handeln so einzurichten, daß Auschwitz nicht sich wiederhole, nichts Ähnliches geschehe." ${ }^{30}$ Nicht nur den Österreichern (oder Deutschen) qua ihrer spezifischen Geschichte, sondern der Menschheit allgemein fällt die Aufgabe zu, etwas Ähnliches wie Ausschwitz zukünftig zu verhindern. Selbst in einem Text, in dem es um die Aufarbeitung der Vergangenheit in Deutschland geht, begrenzte Adorno das Nachleben des Faschismus nicht nur auf jene Länder, in denen er zur Macht kam: „Das faschistische Wunschbild heute verschmilzt ohne Frage mit dem Nationalismus der sogenannten unterentwickelten Länder, die man bereits nicht mehr solche, sondern Entwicklungsländer nennt.“" ${ }^{\text {31 }}$ Zur Macht kam der Nationalsozialismus zwar zuvorderst in Deutschland und schließlich auch in Österreich, Sympathien flossen ihm jedoch aus der ganzen Welt zu. Insofern handelt es sich auch nicht um ein Phänomen, das nur auf Mitteleuropa beschränkt war. Bereits in den 1930erJahren berichtete Alexander Stein von den Versuchen der Nationalsozialisten, in anderen Ländern Fuß zu fassen, und zwar mithilfe des Antisemitismus. Stein war Sozialdemokrat und schrieb sein Buch „Adolf Hitler. Schüler der, Weisen von Zion““ im Exil in Prag. Die Nationalsozialisten betrieben bereits in den 1930ern in Nordafrika, dem arabischen Raum und am Balkan antisemitische Hetzpropaganda. ${ }^{32} \mathrm{Ab}$ den 1940er-Jahren gab es in Berlin Zeesen einen nationalsozialistischen Radiosender, der im arabischen und islamischen Raum Propaganda verbreitete. Geleitet wurde dieser Radio-Sender von Amin El-Husseini, Mufti von Jerusalem, der nach dem Zweiten Weltkrieg dem alliierten Kriegsgericht entkam und seine antisemitische Ideologie nach 1945 gegen Israel fortführen konnte. ${ }^{33}$ Der Antisemitismus ist ein heute globales Problem und war es, wie diese Beispiele zeigen, schon vor und während des Zweiten Weltkriegs. So gab es am

\footnotetext{
28 Szigetvari, Starke antisemitische Vorurteile.

${ }^{29}$ Bundesministerium für Bildung, Hammerschmid: Feindbilder können mit Bildung überwunden werden, 23.11.2017, [https://www.bmb.gv.at/ministerium/vp/2017/20171123.html], eingesehen 11.12.2017.

30 Theodor W. Adorno, Negative Dialektik, Frankfurt a. M. 1975, S. 358.

31 Theodor W. Adorno, Was bedeutet: Aufarbeitung der Vergangenheit, in: Ders., Kulturkritik und Gesellschaft II. Eingriffe Stichworte, Frankfurt a. M. 2003, S. 565.

32 Alexander Stein, Adolf Hitler. Schüler der „Weisen von Zion“, Freiburg i. Br. 2001, S. 149.

33 Vgl. den Beitrag von Michel Wyss in diesem Band.
} 
Balkan und in Osteuropa muslimische SS-Divisionen und Freiwilligenbataillone, die in den Genozid an den europäischen Juden involviert waren. Im Irak kam es 1941 zu einem prodeutschen Putsch und infolgedessen zu einem Pogrom gegen die irakischen Juden. Auch darüber wäre im Geschichtsunterricht zu sprechen. Die Beschäftigung mit dem Nationalsozialismus und dem Holocaust muss sich von ihrer eurozentristischen Sicht lösen. ${ }^{34}$

Es lässt sich kein vernünftiges Argument anführen, warum sich 75 Jahre nach dem Ende des nationalsozialistischen Völkermordes an den Juden heutige Kinder und Jugendliche aufgrund einer spezifischen österreichischen Geschichte in besonderem Maße dafür verantwortlich fühlen sollten. Egal ob es sich dabei um türkische, arabische oder österreichische Jugendliche handelt. Damit keine Missverständnisse aufkommen: Der Kampf gegen und die Aufklärung über den Antisemitismus sind dringend notwendig. Dieser sollte nur nicht aus einer wie auch immer gearteten Verantwortung der Österreicher abgeleitet werden, denn damit wird mit gutem Gewissen der Irrglaube an überzeitliche Kollektive und unveränderliche Volkssubjekte konserviert. Zudem geht es beim Kampf gegen den Antisemitismus nicht um die Imagepflege Österreichs. Stattdessen sollten Schülern die psychosozialen Mechanismen bewusst gemacht werden, die in ihnen selbst den Antisemitismus erzeugen. Um dazu in der Lage zu sein, müssten Lehrkräfte, wie Adorno bereits 1964 vorgeschlagen hat, die gängigsten Propagandatricks der Antisemiten analysieren und den Schülern erklären, warum sie funktionieren und was falsch an ihnen ist. Das wäre insbesondere angesichts der massenhaften Verbreitung und Nutzung von „Sozialen Medien“ notwendig. Vor allem aber muss, so Adorno, das Selbstbewusstsein der Kinder und Jugendlichen gestärkt werden, um den Antisemitismus bekämpfen zu können. ${ }^{35}$

Der Antisemitismus ist nicht nur „das Gerücht über die Juden“, wie es oft aus dem Zusammenhang gerissen zitiert wird. Die antisemitische Ideologie enthüllt auch, was der Antisemit insgeheim wünscht. So definiert Adorno den Antisemiten und seine Wirkung auf andere folgendermaßen: „Paranoia, der Verfolgungswahn, der die anderen verfolgt, auf die er projiziert, was er selber möchte, steckt an."36 Rathkolb übergeht diesen spezifischen Charakter des Antisemitismus und empfiehlt die Dekonstruktion von Vorurteilen ausgehend von selbst erlebten Diskriminierungserfahrungen. Muslimische Jugendliche sollen zuerst über ihre eigene Ausgrenzungserfahrung reden. In einem späteren Schritt soll durch diese Thematisierung der eigenen Ausgrenzung auch Empathie für andere ausgegrenzte Gruppen geweckt werden. Ausgrenzung persönlich

\footnotetext{
34 Nähere Informationen zum Verhältnis von Islam und Nationalsozialismus finden sich in folgenden Werken: Matthias Künzel, Von Zeesen bis Beirut. Nationalsozialismus und Antisemitismus in der arabischen Welt, in: Doron Rabinovici/Ulrich Speck/Natan Sznaider, Neuer Antisemitismus?, Frankfurt a. M. 2004; Jeffrey Herf, Nazi Propaganda for the Arab World, New Haven-London 2009; Klaus-Michael Mallmann/Martin Cüppers, Halbmond und Hakenkreuz. Das Dritte Reich, die Araber und Palästina, Darmstadt 2011.

35 Theodor W. Adorno, Zur Bekämpfung des Antisemitismus heute, in: Ders., Gesammelte Schriften, Band 20, Frankfurt a. M. 1985, S. 371.

36 Adorno, Aufarbeitung, S. 566.
} 
zu erfahren, macht jedoch niemanden zu einem besseren oder reflektierten Menschen. Vermutlich ist eher das Gegenteil der Fall. Die subjektiv erfahrende Ausgrenzung wird selbst oft wieder menschenfeindlich verarbeitet. Davon berichtet auch Adorno in seinem Aufsatz „Zur Bekämpfung des Antisemitismus heute“ von 1964, in dem er sich explizit mit der pädagogischen Praxis im Kampf gegen Antisemitismus auseinandersetzte. Für den Antisemitismus seien vor allem die Gruppen von sehr schlechten Schülern anfällig, analysierte Adorno. Die Lehrpersonen würden auf diesen von vornherein herumhacken. Diesen Schülern würden Fragen in der Erwartung gestellt, dass sie diese nicht beantworten können. Ihre eigene Situation als Stigmatisierte wälzten diese Schüler auf andere, noch schwächere Gruppen ab. Weit entfernt davon dumm zu sein, seien diese Kinder in bestimmten Bereichen sogar sehr begabt. Sie fühlten das Potential in sich. Wenn man diese Kinder nicht mehr durch Erziehung erreiche, weil sich bereits eine autoritäre Charakterstruktur herausgebildet habe, müsse man sie die Autorität spüren lassen. Die Verbreitung des Antisemitismus solle in der Schule unter Strafe gestellt werden. Noch wichtiger aber sei es, diese Kinder zum Sprechen zu bringen. Sie sollten lernen, sich richtig auszudrücken, denn Gefühle benennen zu können und auch auszusprechen ist heilsam. Doch nicht nur deshalb sei diese Maßnahme wichtig: Wer selbst gelernt habe sich gut auszudrücken, werde keinen unbewussten Hass auf redegewandte Kinder ausbilden. ${ }^{37}$ Adornos Beispiele mögen zeitbedingt antiquiert wirken. In der pädagogischen Praxis hat sich seit den 1960er-Jahren wohl einiges geändert, aber Diskriminierungs- und Ausgrenzungserfahrungen - darunter auch durch Lehrpersonen verursachtes Mobbing - sind in der Gegenwart nach wie vor Teil des schulischen Alltags. In ihnen liegt oftmals der Keim für die Ausbildung menschenfeindlicher Ressentiments.

Was Rathkolb wohl implizit kritisieren wollte, ist eine gesellschaftlich weit verbreitete Fremdenfeindlichkeit. Diese richtet sich heute vor allem gegen Migranten und Migrantinnen aus mehrheitlich islamischen Ländern. Die Fremdenfeinde greifen diese Menschen meist pauschal als „Muslime“ an, unabhängig davon, wie es diese Einzelpersonen selbst mit der Religion halten. Gegen diese Fremdenfeindlichkeit die Vorzüge des Islam in Anschlag zu bringen, wäre allerdings nicht nur falsch, sondern aus vielen Gründen kontraproduktiv. Für Adorno war es selbstverständlich, jeder Art von Stereotypenbildung entgegenzutreten. Er warnte deshalb eindringlich davor, negative Gruppenurteile durch positive Gegenbeispiele zu ersetzen. Dagegen wären Urteile über Gruppen von Menschen an sich in Frage zu stellen. ${ }^{38}$ Dem negativen Islambild ein positives Bild entgegenzusetzen, ist selbst wieder eine Stereotypbildung, die Gruppenurteile verfestigt. Zudem werden migrantische Jugendliche aus islamischen Ländern dadurch in Gruppenidentitäten gezwängt. Fatal ist dies deshalb, weil religiöse oder nationale Gruppen die Tendenz haben, sich über den Ausschluss von Fremden oder

37 Ebd., S. 376-377.

38 Adorno, Zur Bekämpfung, S. 378 
Anderen zu definieren. Statt nun das Selbstbewusstsein zu stärken, was geboten wäre, empfiehlt man Kindern und Jugendlichen die Identifikation mit einem Kollektiv. Weder gegen Antisemitismus noch gegen Fremdenfeindlichkeit kann unter diesen Voraussetzungen vorgegangen werden.

Schüler und Schülerinnen müssen auch in diesem Fall völlig unabhängig von ihrer Herkunft im Unterricht über die Mechanismen von Fremdenfeindlichkeit aufgeklärt werden. Die Lehrpersonen sollten sich dabei nicht die Illusion machen, Kinder und Jugendliche, die selbst diskriminiert werden oder wurden, seien von solchen Denkmustern frei. Dies lässt sich auch aus der schon genannten Studie von Güngör ablesen; $20 \%$ der befragten Muslime äußerten sich negativ gegenüber anderen (nichtjüdischen) ethnischen oder religiösen Gruppen.

\section{Was ist Antisemitismus? Die Kritik des Antisemitismus bei Adorno und Horkheimer}

Um dem Antisemitismus entgegentreten zu können, muss man wissen, womit man es zu tun hat. Im April 2017 wurde im österreichischen Ministerrat die Arbeitsdefinition der International Holocaust Remembrance Alliance (IHRA) angenommen. Sie lautet wie folgt:

„Antisemitismus ist eine bestimmte Wahrnehmung von Juden, die sich als Hass gegenüber Juden ausdrücken kann. Der Antisemitismus richtet sich in Wort oder Tat gegen jüdische oder nicht-jüdische Einzelpersonen und/oder deren Eigentum, sowie gegen jüdische Gemeindeinstitutionen oder religiöse Einrichtungen. “39

Wirklich viel schlauer wird man aus dieser Definition allerdings nicht: Antisemitismus wird hier als Hass gegenüber Juden oder deren Institutionen beschrieben. Darauf wäre man wohl auch von selbst gekommen. Deshalb werden der tautologischen Definition insgesamt elf Beispiele für Antisemitismus zur Seite gestellt: Ein Beispiel ist dem christlichen Antisemitismus entlehnt; es ist der Vorwurf an die Juden, Jesus getötet zu haben. Fünf der Beispiele thematisieren die Feindschaft gegen Israel, den zeitgemäßen Ausdruck des Antisemitismus. Die Definition verharrt damit auf einer rein beschreibenden Ebene und lässt jede Erklärung vermissen. Damit bleibt auch völlig unklar, wie sie als Grundlage für eine Pädagogik gegen Antisemitismus dienen könnte. Will man mehr über die Ursache des Antisemitismus erfahren, lohnt ein Blick in die Werke der kritischen Theorie. In Fragen der Pädagogik, dem Nachleben des Faschismus in der Demokratie und der Aufklärung über den Antisemitismus wird gerne auf Theodor W. Adorno verwiesen. Wer sich mit bestimmten Themen beschäftigt, kommt nicht

39 Was ist Antisemitismus. Österreich nimmt IHRA-Arbeitsdefinition von Antisemitismus an, in: erinnern.at, o. D. [http://www.erinnern.at/bundeslaender/oesterreich/e_bibliothek/antisemitismus-1/was-ist-antisemitismus-

oesterreich-nimmt-ihra-arbeitsdefinition-von-antisemitismus-an], eingesehen 11.12.2017. 
darum herum, diesen Autor zu zitieren, gilt er doch als großer Denker. Leider dient dieser Verweis auf Adorno häufig nicht dem Erkenntnis- sondern rein dem Distinktionsgewinn. Unterschlagen wird dabei in der Regel, was Adorno wirklich geschrieben hat und worauf seine Schriften hinauslaufen. Gerade für das Verständnis des Antisemitismus ist dies aber unabdingbar.

Während des Zweiten Weltkriegs verfassten Adorno und Max Horkheimer ihre wohl berühmteste Schrift: Die Dialektik der Aufklärung. Darin findet sich das Kapitel „Elemente des Antisemitismus“. Der Untertitel lautet vielsagend „Grenzen der Aufklärung“. Denn über den Antisemitismus aufzuklären, ist schwierig, entspringt er doch Regungen und Bedürfnissen, die im Unbewussten liegen. Um über die Mechanismen des Antisemitismus aufzuklären, bedienten sich die beiden Autoren der Psychoanalyse von Sigmund Freud. ${ }^{40}$ Nach Freud ist der Mensch nicht der Herr im eigenen Haus, also der Psyche. Die Gründe für das Tun und Handeln der Menschen bleiben ihnen selbst oft verborgen. Doch auch über die Gesellschaft bestimmen die Menschen nicht bewusst, es verhalte sich vielmehr umgekehrt: die Logik der Gesellschaft bestimmt, wie die Menschen agieren. Über die unbewussten Triebkräfte der Gesellschaft und die des einzelnen Individuums wollten Adorno und Horkheimer aufklären. Sie bedienten sich dazu nicht nur Freud, sondern auch der Kritik der politischen Ökonomie von Karl Marx. ${ }^{41}$

Der Antisemitismus hat für Adorno und Horkheimer ökonomische und psychologische Gründe und dies gleichzeitig. Gerade von marxistischer Seite gab es immer wieder Versuche, den Antisemitismus rein ökonomisch zu erklären. Das musste misslingen. Denn die Antisemiten folgten keinen rationalen ökonomischen Zielen. Vom deutschen Beamten bis zum Bewohner von Harlem wüssten alle Antisemiten, so schrieben Horkheimer und Adorno in den 1940ern, dass er sich letztlich nicht auszahle. Doch der Gedanke daran, andere hätten es noch schlechter, reicht dem Antisemiten als Motivation. Der Antisemitismus zielt nicht auf die Verbesserung der materiellen Lage von einzelnen Menschen, er zielt ausschließlich auf die Vernichtung der Juden. ${ }^{42}$

Marx und Freud zusammendenken heißt: Die Psychoanalyse muss den Menschen untersuchen, der unter bestimmten ökonomischen Verhältnissen lebt. Verändern sich die ökonomischen Verhältnisse, verändern sich auch die Charaktere jener Menschen, die in diesen leben. Deshalb sind Erkenntnisse, die Freud im frühen 20. Jahrhundert machte, an die spezifischen gesellschaftlichen und ökonomischen Bedingungen der damaligen Zeit in Wien geknüpft. Das Über-Ich ist bei Freud jene Instanz im Menschen, die über Verbote, Gebote und Werte des Individuums wacht. Dieses Über-Ich bildete sich im 19. Jahrhundert in der Auseinandersetzung mit dem Vater aus. Dies hatte ein ökonomisch starkes und autonomes Bürgertum zur Voraussetzung. Denn nur unter diesen

$40 \mathrm{Zu}$ den Verbindungen der beiden Ansätze siehe auch: Christine Kirchhoff/Falko Schmieder (Hrsg.), Freud und Adorno. Zur Urgeschichte der Moderne, Berlin 2015.

${ }^{41}$ Karl Max, Zur Kritik der Politischen Ökonomie [1859] (Marx-Engels-Werke 13), Berlin 1971.

42 Horkheimer/Adorno, Dialektik der Aufklärung, S. 199. 
Verhältnissen konnte der Vater als stark und autonom erscheinen. Dies galt nur in der liberalen Phase des Kapitalismus. Freud erlebte in Wien gerade noch dessen Ausläufer mit. Im Spätkapitalismus bildet sich das Über-Ich nicht mehr am Vater, sondern an gesellschaftlichen Institutionen wie Schule, Sportverein, Arbeit oder Peer-Groups. Diese haben zu einer radikalen Veränderung des aktuellen Menschentypus geführt. Diese hierarchische Gesellschaftsform, welche sich in Institutionen und der Selbstzurichtung der Subjekte äußert, müsse die Menschen irrational machen. ${ }^{43}$

Für den Antisemitismus heißt dies nun: Er hat ökonomische Gründe, die mithilfe der Psychoanalyse besser verstanden werden können. Wünsche und Regungen im Einzelnen, die gesellschaftlich tabuisiert sind, werden jemand anderem zugeschrieben.

Nach Adorno und Horkheimer produziert die falsch eingerichtete Gesellschaft den Vernichtungswillen gegen die Juden aus sich heraus. ${ }^{44}$ Die ökonomische Ordnung verdamme die Mehrheit der Menschen zur Abhängigkeit von Bedingungen, über die sie nicht verfügten. Damit aber nicht genug: Um in dieser Ordnung leben zu können oder gar erfolgreich zu sein, müssen sich die Menschen anpassen. Ihre Autonomie wird dabei durchgestrichen, also genau jener Moment, an den die Demokratie appelliert. Dem zu widerstehen verlangt neben einer Ich-Stärke auch die Kraft, nicht um jeden Preis mitmachen zu wollen. Verstärkt wird das totalitäre Potential durch die Wut und Unzufriedenheit, die der schmerzhafte Zwang zur Anpassung an Verhältnisse produziert, die nicht glücklich machen. Weil der autoritäre Charakter realisiert, dass er selbst in der Anpassung um das versprochene Glück der Gesellschaft betrogen wurde, hasst er nun jeden Gedanken an eine Welt, in der das Glück möglich wäre. Diese Sehnsucht nach Glück, Einkommen ohne harte Arbeit, eine Gesellschaft ohne Druck verschwindet im Einzelnen nicht einfach. Diese Regungen werden verleugnet und den Juden zugeschrieben. Alles was die Antisemiten insgeheim wünschen, lasten sie den Juden an: Sowohl den Reichtum, der ihnen vorenthalten bleibt, die sexuelle Freizügigkeit als auch den Gedanken an eine befreite Gesellschaft. Für die Anpassungsleistung an die falsche Gesellschaft wollen die Betrogenen belohnt werden, zwar nicht mit Reichtum, aber mit der Zusage, ewig ausgebeutet zu werden. Insgeheim weiß der Betrogene, wie überflüssig und austauschbar er in der Arbeit ist. Deshalb fordert er das Vorrecht der Nationalität und argumentiert mit der Herkunft. Die Gesellschaft, die er selbst nicht durchschauen will, weil dies ihm die Nutzlosigkeit seiner Anpassung vor Augen führt, muss um jeden Preis affirmiert werden. Für das Schlechte auf der Welt sind nicht die elenden Verhältnisse verantwortlich, sondern bestimmte gierige Menschengruppen. Dies ist der gesellschaftliche und ökonomische Grund des Antisemitismus. ${ }^{45}$

In der Unterdrückung von Wünschen liegt auch der Grund für den islamischen Antisemitismus begraben. Der Islam verlangt dem Namen nach Unterwerfung und

43 Max Horkheimer, Der soziologische Hintergrund des psychoanalytischen Forschungsansatzes, in: Ernst Simmel (Hrsg.), Antisemitismus, Frankfurt a. M. 1993, S. 32-33.

${ }^{44}$ Horkheimer/Adorno, Dialektik der Aufklärung, S. 197.

45 Adorno, Aufarbeitung, S. 560-567. 
zwingt seinen Anhängern den Triebverzicht auf. Der Westen führt sie nun immerzu in Versuchung, in Sünde zu leben, die sie selbst begehren, sich jedoch nicht eingestehen dürfen. Ansonsten würden sie vom wahren Glauben abkommen. Gehasst werden nun jene, die für gesellschaftliche Libertinage stehen. Es ist das Bild des Juden, der hinter den Verführungen des Westens stehen soll. Auch der islamische Antisemit hasst seine eigenen Wünsche.

\section{Wie gegen Antisemitismus vorgehen?}

In Graz beschäftigte sich der Verein JUKUS - Verein zur Förderung von Jugend, Kultur und Sport im Jahre 2017 mit dem Thema Antisemitismus. Zu diesem Zweck wurde auch eine Broschüre mit dem Namen ,Jugend, Migration und Antisemitismus. Präventive Arbeit zu menschenfeindlichen Haltungen "veröffentlicht. ${ }^{46}$ Diese Broschüre nimmt sich explizit dem Thema des Antisemitismus unter Migranten an. Dieser äußere sich vielfältig: Israel oder die Juden insgesamt werden als Feinde gesehen. Das Wort ,Jude“ wird auf dem Schulhof als Schimpfwort verwendet. ${ }^{47}$ Manche Jugendliche vertreten sogar die Position, die Muslime und die Juden seien schon immer Feinde gewesen. Womit sie sich auf einige Koranstellen stützen können, die von der Vernichtung jüdischer Stämme durch Mohamed berichten. ${ }^{48}$ Auch der Nationalsozialismus versuchte bei seiner Propaganda in der islamischen Welt an diese Argumentationsfigur anzuschließen.

Die meisten muslimischen Jugendlichen wurden in Österreich selbst schon diskriminiert, betont die Autorin. Auch in dieser Broschüre empfiehlt man, an diesen Erfahrungen anzuknüpfen. Wer selbst nicht gerne ausgegrenzt wird, der wird besser verstehen, warum er selbst niemand ausgrenzen soll, so zumindest der Gedanke hinter diesem Ansatz. Bei Jugendlichen, die für Erfahrung noch offen sind, kann dies sogar funktionieren. Doch wahrscheinlich waren genau diese Jugendliche, bei denen es noch funktioniert, gar nie anfällig für die antisemitische Ideologie. Wer an Unterdrückungserfahrungen anschließt, läuft oft Gefahr eine gewisse Opfer-Identität zu stärken. Diese Gefahr darf keinesfalls unterschätzt werden. Wer sich als Opfer übermächtiger Gegner empfindet, fühlt sich zum besinnungslosen Zuschlagen legitimiert. An den nichtigsten Ereignissen entlädt sich dann die Gewalt. Ob der Anlass nun die Verlegung der US-Botschaft nach Jerusalem oder die Veröffentlichung von Mohammed-Karikaturen in dänischen Zeitungen ist.

Allgemein wird in der Broschüre die Wirkmächtigkeit von Erziehung und Pädagogik überschätzt. Adorno reflektiert auf diese Grenze der Pädagogik und gibt einige praktische

\footnotetext{
${ }^{46}$ Johanna Stadelbauer, Jugend, Migration und Antisemitismus. Präventive Arbeit zu menschenfeindlichen Haltungen, Graz 2017, [http://jukus.at/sites/default/files/uploads/broschuere_web.pdf], eingesehen 1.6.2020.

47 Siehe dazu auch Franziska Pröll, Wenn Schüler einander als „Du Jude!“ beschimpfen, in: Die Zeit, 21.12.2019, [https://www.zeit.de/gesellschaft/schule/2019-12/antisemitismus-beleidigung-schulhof-paedagogik-lehrerausbildung], eingesehen 1.6.2020.

48 Vgl. den Beitrag von Michel Wyss in diesem Band.
} 
Ratschläge, wie gegen den Antisemitismus vorzugehen sei. Zuerst müsse erforscht werden, wo und wie der faschistische Antisemitismus nachlebt. Diese Forschungsarbeit werde vom Gedanken geleitet, die Mechanismen des Antisemitismus zu begreifen und darauf zu reflektieren. Der falsche Weg sei dagegen, sich ausschließlich moralisch über den Antisemitismus und die Antisemiten zu entrüsten. ${ }^{49}$

Für Adorno muss auf zwei verschiedene Arten gegen Antisemitismus vorgegangen werden. Da wären die Langzeitprogramme, die durch Erziehung verhindern sollen, dass Kinder einen autoritätsgebundenen Charakter ausbilden. Doch ab einem gewissen Alter seien die Würfel in diese Richtung gefallen und mit Erziehung und Pädagogik ist dem Antisemitismus nicht mehr beizukommen. Deshalb empfiehlt Adorno Kurzzeitprogramme zur akuten Abwehr des Antisemitismus. Diese Programme können auf Autorität nicht verzichten. ${ }^{50}$ Das mag widersprüchlich erscheinen. Auf der einen Seite soll die Herausbildung autoritärer Charakterstrukturen durch Erziehung verhindert werden, auf der anderen Seite soll man gegen den Antisemitismus mit autoritären Mitteln vorgehen. Adorno plädiert nun dafür, Phänomene nicht dort zu vereinfachen, wo die Realität den Menschen Widersprüche aufzwingt. In der Erziehung muss der Herausbildung eines autoritätsgebundenen Charakters entgegengearbeitet werden. Abgebaut werden soll jede Autorität, die sich nicht durch Vernunft oder Sachautorität legitimiert lässt. ${ }^{51}$ Dies ist ein erster Schritt zur Entbarbarisierung. Denn der autoritäre Charakter ist anfällig für das antisemitische Vorurteil. Wenn sich dagegen nichts mehr machen lässt, weil die Charakterstruktur sich schon zu einer autoritären verfestigt hat, bleibt als Mittel gegen den Antisemitismus nur mehr die Autorität. Denn diese Personen sind für Erfahrung nicht mehr offen, sie lassen sich nicht mehr einfach so verändern. ${ }^{52}$ Adorno berichtete in einer längeren Passage von einem Aufeinandertreffen mit Antisemiten:

„Ich sagte Ihnen bereits, dass ich bei Menschen, bei denen das Vorurteil bereits etabliert ist, von der Herstellung sogenannter Kontakte und ähnlichem nicht viel halte. Bei ihnen ist die Erfahrungsfähigkeit bereits abgestumpft. Antisemitischen Äußerungen ist sehr energisch entgegenzutreten: sie müssen sehen, dass der, welcher sich gegen sie stellt, keine Angst hat. Man imponiert einem bissigen Hund, sobald er merkt, dass man sich nicht vor ihm fürchtet, aber es ist verloren, wenn er innerviert, dass man eigentlich vor seinem Gebiss zittert; so ist es in solchen Fällen auch. Ich habe nach meiner Rückkehr nach Deutschland mit solchen Menschen unmittelbare Erfahrung gemacht. Einmal bin ich an einer Gruppe von Chauffeuren vorbeigekommen, die damals in dem Pool für die amerikanische Besatzungsmacht beschäftigt waren. Sie schimpften untereinander wüst auf die Juden. Ich ging zum nächsten Schutzmann und ließ sie verhaften. Auf der Wache habe ich mich lange und eingehend vor allem mit dem Rädelsführer unterhalten und habe

49 Adorno, Bekämpfung, S. 362.

50 Ebd., S. 372.

51 Theodor W. Adorno, Erziehung zur Mündigkeit, Frankfurt a. M. 1971, S. 131.

52 Adorno, Bekämpfung, S. 363-364. 
von ihm einen Satz gehört, der sich mir sehr eingeprägt hat: ,Ach wissen Sie, gestern waren wir Nazi, heute sind wir Ami und morgen sind wir Kommi` Er hat mir damit ungewollt eine tiefe Weisheit über die ganze Charakterstruktur seines Typus verraten. Bei ihm überwiegt das Motiv der Anpassung um jeden Preis alles andere. Wenn man in solchen Fällen ohne Angst zugreift und dann auf die Argumente solcher Personen in allem frank antwortet, kann man etwas erreichen. Ich hatte jedenfalls das Gefühl, dass jene Chauffeure, jedenfalls ihrer bewussten Überzeugung nach, ein wenig anderen Sinnes von der Polizeiwache weggegangen sind. Begegnet man expliziten und fixierten Vorurteilen, so ist auf eine Art Schocktherapie zu vertrauen. Man muss die allerschroffsten Gegenpositionen beziehen. Schock und moralische Kraft gehen dabei zusammen. Schlecht ist das Zurückweichen. "53

Allgemein war Adorno kein Befürworter unreflektierter antiautoritärer oder pazifistischer Erziehung. Auch wenn Kinder dazu erzogen werden sollten, Abscheu gegenüber jeder Art von psychischer Gewalt zu empfinden ${ }^{54} \mathrm{zu}$ „Lämmchen“ sollen sie keinesfalls werden. Denn „Lämmchen“ wären wahrscheinlich bereit, so Adorno, das Abscheuliche ohne Gegenwehr hinzunehmen. ${ }^{55}$

\section{Aufklärung statt Propaganda}

Wer gegen Antisemitismus vorgeht, muss auf alles reklameähnliche verzichten, so die Forderung Adornos. Stattdessen müssten Kinder und Jugendliche immer mit der ungeschönten und ganzen Wahrheit konfrontiert werden. Aufgeklärt werden muss vor allem über die Struktur und die Motivation des antisemitischen Denkens. Wer sich dagegen auf Diskussionen innerhalb einer antisemitischen Argumentation einlässt, also antisemitische Behauptungen mit Gegenbehauptungen kontert, steht schon von Beginn an auf verlorenem Posten. Wer nun bspw. als Lehrperson mit der Behauptung konfrontiert wird, am 11. September 2001 seien gar keine Juden in den New Yorker Twin Towers gewesen, weil sie wohl selbst die Drahtzieher hinter den Anschlägen waren, sollte sich gar nicht erst auf Zahlenspiele einlassen. Offenzulegen wäre hier vielmehr die zugrundeliegende Struktur des antisemitischen Verschwörungsdenkens. Die pathische Projektion, die den Juden ein Verbrechen andichtet, welches von Anhängern des Islam verübt wurde (und von diesen auch durchaus für sich reklamiert wurde). Derartige Projektionen können mehrere, sich überlagernde Gründe haben: Man will beweisen, dass auch die Juden bösartig sind, womit der Antisemitismus der Nazis im Nachhinein legitimiert oder relativiert werden soll (sogenannter ,sekundärer Antisemitismus“56).

53 Ebd., S. 379-380.

54 Adorno, Erziehung, S. 130-131.

55 Ebd., S. 128-129.

56 Juliane Wetzel, Antisemitismus heute - klassische und neue Erscheinungsformen, in: bpb.de, 23.11.2017, [https://www.bpb.de/politik/extremismus/rechtsextremismus/260281/antisemitismus-heute-klassische-und-neue- 
Oder aber es wird als Plan der Juden verkauft, den Islam schlecht dastehen zu lassen und die USA in einen Krieg gegen islamische Länder zu zwingen. Muslime könnten dies nämlich nie tun, für sie sei es verboten Unschuldige zu töten usw.

Ein anderes Beispiel, das Adorno selbst anführte, stammt noch aus dem Arsenal des nationalsozialistischen Antisemitismus. Die Behauptung, die Juden drückten sich vor harter Arbeit. Dem entgegen zu halten, viele Juden in Osteuropa seien Arbeiter gewesen, ist für Adorno der falsche Weg. Dagegen sollte offengelegt werden, was hinter diesem Vorwurf eigentlich steckt: Die eigene uneingestandene Abscheu gegen die Arbeit. Diese darf man sich nicht offen eingestehen, verlangt doch die Gesellschaft von jedem Einzelnen, dass er hart arbeitet. Deshalb hasst man jene, die angeblich schon ohne harte Arbeit leben: Die Juden. Die einzige richtige Entgegnung wäre hier für Adorno: Harte Arbeit alten Stils sei heute gar nicht mehr nötig. Dank der Entwicklung der Wissenschaft, der Technik und der Maschinen könnte man die harte Arbeit von heute auf morgen abschaffen. ${ }^{57}$ Auch dem Antisemitismus soll kein Idealbild des Juden gegenübergestellt werden. Der Antisemitismus entspringt den Projektionen der Antisemiten. ${ }^{58}$ Wer gegen den Antisemitismus vorgehen will, muss immer bei der Wahrheit bleiben. Deshalb wäre es auch nicht die richtige Strategie, den jüdischen Beitrag zum kulturellen und politischen Leben der Weimarer Republik zu leugnen. Auch wer sich auf diese Diskussion einlässt, hat verloren. In einer liberalen Demokratie verletzt schon diese Diskussion darüber, welche Bevölkerungsgruppen in welchen Berufen überrepräsentiert seien, das Gesetz der Gleichheit. ${ }^{59}$

Wie nun gegen den Antisemitismus vorgehen? Auch wenn sie gut gemeint sind, für Adorno sind Freundschaftsprogramme zwischen Juden und Nicht-Juden im Kampf gegen Antisemitismus meist wirkungslos. Die Antisemiten sind zu keiner Erfahrung fähig. Sie können deshalb nicht durch Begegnungsprogramme mit Juden von ihrem Antisemitismus abgebracht werden. Die Geschichte des Antisemitismus zeigt, dass Begegnungen zwischen Juden und Nicht-Juden letztlich eben diesen nicht verhinderten. Auch die Präsentation von Fakten wird im Kampf gegen den Antisemitismus zahnlos bleiben. Stattdessen muss man den Einzelnen bewusstmachen, welche Mechanismen den Antisemitismus vielleicht auch in ihnen selbst erzeugen. ${ }^{60}$ Und wenn dies alles nichts mehr hilft, muss entschieden gegen den Antisemitismus vorgegangen werden, wenn nötig auch mit den Mitteln der Autorität.

erscheinungsformen-einer-ideologie], eingesehen 1.12.2019.

57 Adorno, Bekämpfung, S. 369-370.

58 Ebd., S. 369.

${ }^{59}$ Ebd., S. 362-363.

60 Ebd., S. 371. 